\title{
Randomized Mechlorethamine/Chlormethine Induced Dermatitis Assessment Study (MIDAS) Establishes Benefit of Topical Triamcinolone 0.1\% Ointment Cotreatment in Mycosis Fungoides
}

\author{
Carolina V. Alexander-Savino • Catherine G. Chung • Elaine S. Gilmore •
}

Sean M. Carroll · Brian Poligone (D)

Received: November 24, 2021 / Accepted: January 8, 2022 / Published online: February 5, 2022

(C) The Author(s) 2022

\begin{abstract}
Introduction: Treatment of early-stage mycosis fungoides (MF) requires safe, skin-directed therapies. Medication side effects can lead to underutilization of effective therapies. The objective of this study was to assess the use of topical triamcinolone $0.1 \%$ ointment as a means of reducing contact dermatitis associated with topical mechlorethamine/chlormethine gel for the treatment of MF.

Methods: This prospective, randomized, openlabel study evaluated 28 adults with mycosis fungoides who were eligible for treatment with topical mechlorethamine/chlormethine gel from December 17, 2017 to December 23, 2020. Patients were treated for 4 months with clinical
\end{abstract}

Supplementary Information The online version contains supplementary material available at https:// doi.org/10.1007/s13555-022-00681-6.

C. V. Alexander-Savino · E. S. Gilmore ·

S. M. Carroll · B. Poligone $(\square)$

Rochester Skin Lymphoma Medical Group, 6800

Pittsford Palmyra Rd, Suite 150, Fairport, NY 14450,

USA

e-mail: bpoligone@roclymphoma.com

C. G. Chung

Dermatology and Pathology, The Ohio State

University, Columbus, $\mathrm{OH} 43210$, USA

B. Poligone

Rochester General Hospital Research Institute,

Rochester, NY 14621, USA follow-up through 12 months. Patients had half of their lesions also treated with topical triamcinolone $0.1 \%$ ointment (while the other half were treated with mechlorethamine/chlormethine alone). The study was self-controlled with separate lesions in the same patient receiving each treatment arm. Treatment arms were determined by the flip of a coin.

Results: Twenty-eight patients enrolled (17 men (61\%) and 11 women (39\%)). Demographics included 25 White, 2 African Americans, and 1 Asian patient. Twenty-five completed the 12-month follow-up. Triamcinolone $0.1 \%$ ointment led to increased tolerability of mechlorethamine/chlormethine gel but did not change the efficacy of mechlorethamine/chlormethine. There was a statistically significant $50 \%$ decrease in dermatitis (SCORD score) at month 2 in the triamcinolone-treated arm.

Conclusions: Topical triamcinolone ointment is a helpful adjuvant therapy when treating patients with topical mechlorethamine/ chlormethine gel. It diminishes inflammation and does not reduce efficacy. The peak incidence of dermatitis in the study occurred in the second and third months.

Trial Registration: ClinicalTrials.gov identifier, NCT03380026. 
Keywords: Contact dermatitis; Drug reactions; Lymphoma; MF; Patch testing; Prescription drug management; Therapeutics

\section{Key Summary Points}

Topical mechlorethamine/chlormethine has been effectively used for the treatment of mycosis fungoides for decades

However, use is limited because of early discontinuation, generally due to contact dermatitis

Improved understanding of this drug side effect will support physicians and patients when using this central skin-directed therapy

\section{INTRODUCTION}

Mycosis fungoides (MF) is the most common type of cutaneous $\mathrm{T}$ cell lymphoma. The most common presentation of MF is at early stage with an indolent skin eruption that does not involve the lymph nodes, blood, or viscera [1]. The treatment of early-stage MF requires skindirected therapies, with topicals and ultraviolet light phototherapy the most commonly recommended in current treatment guidelines [2-4]. While overall response rate among therapies is a major comparator, for a disease that is incurable, it often requires extended and even chronic therapy. Therefore, other factors beyond efficacy drive therapy utilization. Recently, by examining the usage of topical mechlorethamine/chlormethine gel among US physicians, we identified that there was a high frequency of discontinuation of therapy after 1 month in providers who had fewer individual patients treated with mechlorethamine/ chlormethine [5]. Underutilization of topicals could lead to increased utilization of more expensive and less safe alternatives.

Topical mechlorethamine (also known as chlormethine) gel therapy was approved by the US Food and Drug Administration (FDA) in
2013 for treating stage IA and IB MF in patients who received prior skin-directed therapy [6]. The same gel formulation has been registered in Israel since 2016 (for the same indication as in the USA) and was approved by the European Medicines Agency in 2017 for treatment of adult patients with MF (Ledaga SmPC). While newly approved therapies often require time for physicians to feel comfortable with both the safety and efficacy, in this case, mechlorethamine/chlormethine has been a mainstay of therapy for early-stage MF for decades. Indeed, the first report of the effects of mustard gas on blood cells was in 1919 [7]. This led to topical mechlorethamine/chlormethine preparations [8]. In an early prospective clinical study examining the efficacy of topical mechlorethamine/chlormethine, Van Scott et al. showed a $79 \%$ complete response rate in plaque-stage disease [9]. In a retrospective study of patients treated at a center of excellence, complete response rates for patch disease were $65 \%$ and an overall response rate of $93 \%$ was observed with topical mechlorethamine/ chlormethine treatment [10]. Thus there are strong data over several decades supporting the efficacy of topical mechlorethamine/chlormethine in the treatment of MF.

Despite its established high efficacy, our recent data showed that $80 \%$ of clinicians, with a low treatment volume of patients with MF, had early discontinuation of topical mechlorethamine/chlormethine (i.e., had only one or two prescriptions filled) [5]. Whereas, providers with more than 15 patients with MF had a median of 6.3 prescriptions dispensed per patient. Prior to the development of mechlorethamine/chlormethine compounded in ointment or gel formulations, mechlorethamine/ chlormethine was distributed as an aqueous solution associated with significant contact dermatitis [11-14]. Although the ointment and gel formulations have decreased the incidence of contact dermatitis, its historical significance likely resonates with some prescribing dermatologists, who may be inclined to discontinue treatment with early signs of dermatitis even with newer preparations. Even with the ointment and gel formulations, contact dermatitis occurs in approximately $15 \%$ of patients and up 
to $50-60 \%$ if symptoms of skin irritation and erythema are included [6]. Importantly, when evaluating 52 weeks of treatment data for the gel and ointment therapies, it is clear that most patients must remain on mechlorethamine/ chlormethine for more than 1 or 2 months to achieve an optimal response. Addressing concerns of contact dermatitis early in the treatment course is therefore paramount to maximizing potential benefit for patients prescribed topical mechlorethamine/ chlormethine.

To address this, some groups have recommended decreased frequencies of application such as twice weekly, or titrated frequency of use $[15,16]$. These recommendations may decrease adverse effects, but might, unfortunately, also lead to decreased efficacy or slower treatment responses.

It is important to note that not all dermatitic reactions associated with topical mechlorethamine/chlormethine represent classic allergic contact type IV delayed hypersensitivity reactions (DHR), which could be expected to worsen with continued exposures. While DHRs are a known possibility with mechlorethamine/ chlormethine [9, 14, 17], a large spectrum of non-DHR cutaneous reactions have also been reported, including irritant contact dermatitis, bullous reactions, and burning sensation $[6,10,11]$. Better understanding and education on potential adverse cutaneous reactions to current topical mechlorethamine/chlormethine formulations may lead to better compliance.

The primary endpoint of the present study was to examine the ability of topical triamcinolone ointment to prevent dermatitis associated with topical mechlorethamine/ chlormethine gel. By adapting an established scale (SCORAD) for measuring dermatitic reactions, the dermatitis could be quantified. Secondary endpoints include examining the variety of dermatitides through pathologic evaluation, patch testing, and examination of $\mathrm{T}$ cell clones/diversity. An additional endpoint compared the efficacy of both arms of treatment through measurement of the composite assessment of index lesions (CAILs) and genetic T cell clonality.

\section{METHODS}

This was a prospective, randomized, controlled study approved by the WCG Institution Review Board. This study was performed in accordance with the Helsinki Declaration of 1964 and its later amendments. All patients voluntarily signed an informed consent form (ICF) that was approved by the Western Institutional Review Board (WIRB). The ICF was delivered verbally by the investigator and/or clinical coordinator and all questions answered by the investigator or coordinator. Consent was provided before any study-related procedures were performed. Adult patients presenting to the Rochester Skin Lymphoma Medical Group, a specialty treatment center for skin lymphoma in Fairport, New York from December 2017 through December 2020, who had early-stage MF (stage IA or IB), were candidates for topical mechlorethamine/ chlormethine and voluntarily agreed to participate in a clinical study were recruited. Of the 29 patients enrolled, 28 met inclusion criteria; among them, 26 patients completed at least 5 months of study, which included the full course of therapy and a month of follow-up. A total of 25 patients completed the 12-month clinical follow-up. Baseline demographics are shown in Supplemental Table S1.

Patients were required to have at least two similar MF lesions based on amount of scale, erythema, elevation, and size. Lesions had to cover at least $8 \mathrm{~cm}^{2}$ in aggregate size. Lesions were separated into two groups for the two treatment arms ensuring similar surface area on each treatment arm. Lesions were also separated by the right side versus the left side of the body, when possible, to facilitate patient compliance. Both arms were treated concurrently in the same patient after at least a 3-week washout period from any previous therapy.

All lesions (i.e., both study arms) were treated with topical $0.106 \%$ mechlorethamine/ chlormethine gel once daily per the FDA-approved prescriber information for 4 months. Half of these lesions were also treated with topical triamcinolone $0.1 \%$ ointment once daily for 4 months. A moderate to high potency topical corticosteroid was selected instead of an 
ultra-high potency corticosteroid because the main goal was to control contact dermatitis rather than to maximally remit disease. At each visit, patients confirmed they were applying the prescribed therapy to the correct lesions. Patients were followed monthly for 5 months and once at 12 months. Skin biopsies were performed at pre-treatment baseline and at 5 months from each treatment arm. In cases of severe cutaneous reactions requiring alteration of the schedule of mechlorethamine/chlormethine application, skin biopsies and patch testing were performed. A composite assessment of indexed lesions (CAILs) was performed monthly.

At each visit a SCORD score, adapted from the Scoring Atopic Dermatitis (SCORAD) tool, was obtained. Each study arm had a SCORD score (vSCORD = mechlorethamine/chlormethine gel only and tSCORD = mechlorethamine/chlormethine gel plus triamcinolone ointment) at each visit. For each score, the surface area of all lesions treated in one therapeutic arm was considered 100\%. The average intensity for redness, swelling, oozing/ crusting, scratch marks, skin thickening, and dryness were measured on a scale from 0 to 3; 0 being none and 3 being severe. Each patient assesses pruritus and sleep disturbance using a visual analog scale (VAS).

Severe dermatitis was defined as blistering, ulceration, and pain or requiring a discontinuation/pause of therapy due to symptomatic contact dermatitis. Patients with severe dermatitis underwent a skin biopsy and patch testing. Mechlorethamine/chlormethine gel was placed in Finn chambers at concentrations (per volume: volume with vehicle alone) of $100 \%, 75 \%, 50 \%, 25 \%$, and vehicle alone. Additionally, the T.R.U.E. Test was performed (SmartPractice, Phoenix, AZ). Assessments were made at 48 and $96 \mathrm{~h}$. Skin biopsies were analyzed by a dermatopathologist (C.C.) blinded to both patient and treatment arm.

Lastly a biopsy was obtained at baseline and at the end of treatment in order to assess the exploratory endpoint of response to therapy and molecular clonality. These biopsies were placed in RNAlater from Thermo-Fisher Scientific (Waltham, MA). Genomic DNA was obtained using Qiagen's DNeasy Blood and Tissue Kit (Germantown, MD) and run on the hsTCRB v4 assay at Adaptive Biotechnologies (Seattle, WA).

\section{Statistical Analysis}

Prior to the study, the number of patients needed to treat to achieve a statistically significant result was calculated to be 22 patients for an $83 \%$ power. This assumes that $10 \%$ of patients treated with topical mechlorethamine/ chlormethine plus triamcinolone versus $56 \%$ of patient treated with topical mechlorethamine/ chlormethine develop dermatitis. This calculation also considers that $89 \%$ of subjects experience contact dermatitis within 4 months of treatment. To account for potential participant withdrawal, a total of 28 patients were recruited and 26 completed the primary endpoint.

A two-tailed $t$ test was performed to determine the significance of the results comparing the incidence of contact dermatitis and for response rates with CAILs between the two groups.

\section{RESULTS}

\section{Contact Dermatitis}

\section{SCORD Scoring}

For this study we developed an adapted SCORAD assessment tool to measure contact dermatitis (Supplemental Fig. S1). The investigator performed a global assessment of each subject's dermatitis as to none, mild, moderate, or severe. Severe dermatitis was when blistering, erosions, or sufficient pain or itch leading to discontinuation of therapy occurred. The SCORD score provides an assessment of dermatitis severity similar to the SCORAD for atopic dermatitis [18] (Fig. 1A). A valid scoring tool should differentiate clinical dermatitis severity. vSCORD is for the topical mechlorethamine/ chlormethine only arm and tSCORD is for the topical mechlorethamine/chlormethine plus triamcinolone arm. Within each group (vSCORD and tSCOD) in patients $(n=27)$ 
assessed as no to mild dermatitis $(n=11)$ compared to moderate to severe dermatitis $(n=16)$, there was a statistically significant $(p<0.05)$ difference in SCORD score after 2 months ( mean $=7.65$, s.d. $=8.15$ versus mean $=13.82$, s.d. 15.82) of treatment and lasting through the end of treatment (month 4). The vSCORD showed a statistical difference in all months where moderate to severe dermatitis was observed (no one had moderate to severe dermatitis after only 1 month of therapy) (Fig. 1B). Therefore, the SCORD scoring is a valid tool to assess dermatitis severity.

During all months of treatment, the addition of triamcinolone ointment (tSCORD) led to a decreased SCORD score relative to the mechlorethamine/chlormethine only arm (vSCORD) (Fig. 2A). The maximum SCORD scores (i.e., the most severe dermatitis) were observed after 2 and 3 months of therapy (month 2: vSCORD max 73.3 versus tSCORD max 59.8; and month 3: vSCORD $\max 72.85$ versus tSCORD max 55.5), which was also the time of maximum benefit of triamcinolone. There was a statistically significant difference at the end of the month 3 between lesions which had received triamcinolone (tSCORD) and those which had not (vSCORD) (Fig. 2B). In subgroup analysis comparing the vSCORD to tSCORD scores during treatment time points, examining

\section{A. SCORD (SCORing Dermatitis)}

A. The percentage of treated lesions that showed dermatitis was calculated. ( 0 represents no dermatitis in treated lesions; 100 represents all treated lesions are entirely involved with dermatitis entirely covers). $A=0$ to 100 .

B. Lesions with dermatitis were measured on a 0-3 point scale (0-None, 1-Mild, 2-Moderate, 3Severe) for a) redness, b) swelling, c) oozing/crusting, d) scratch marks, e) skin thickening, d) dryness. $\mathrm{B}=0$ to 18

C. Patients self-reported their current itch on a 0 to 10 scale by marking itch intensity on a visual analog scale. A slash at 2 centimeters represents and itch score of 2.0. $\mathrm{C}=0$ to 10 .

Calculation of SCORD score $=(A / 5+7 B / 2+C)$, as derived from the established SCORAD scale.

B. Timing of incidence of dermatitis in mechlorethamine/chlormethine gel treated participants showing "None to Mild Dermatitis" vs "Moderate to Severe Dermatitis", according to the investigator's global assessment of dermatitis. Student's t-test, ${ }^{*} p<0.05,{ }^{* *} p<0.01$

$p$ value

\begin{tabular}{|l|l|}
\hline Month 0 & 0.660 \\
\hline Month 1 & 0.246 \\
\hline Month 2 & $0.010^{*}$ \\
\hline Month 3 & $0.001^{* *}$ \\
\hline Month 4 & $0.002^{* *}$ \\
\hline Month 5 & 0.729 \\
\hline Month 12 & 0.958 \\
\hline
\end{tabular}

Fig. 1 A Description of SCORD scoring. B Timing of contact dermatitis reactions measured with SCORD in patients treated with mechlorethamine/chlormethine gel only and clinically presenting with "none to mild dermatitis" versus "moderate to severe dermatitis" 
A.

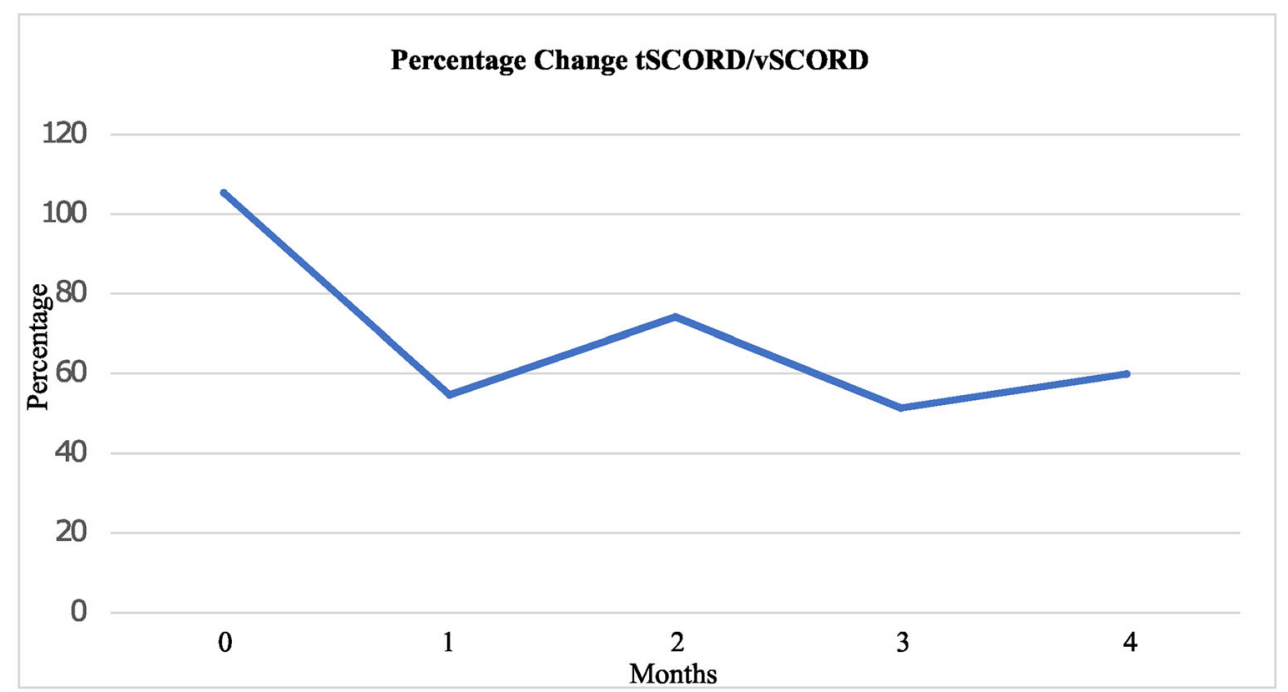

B.

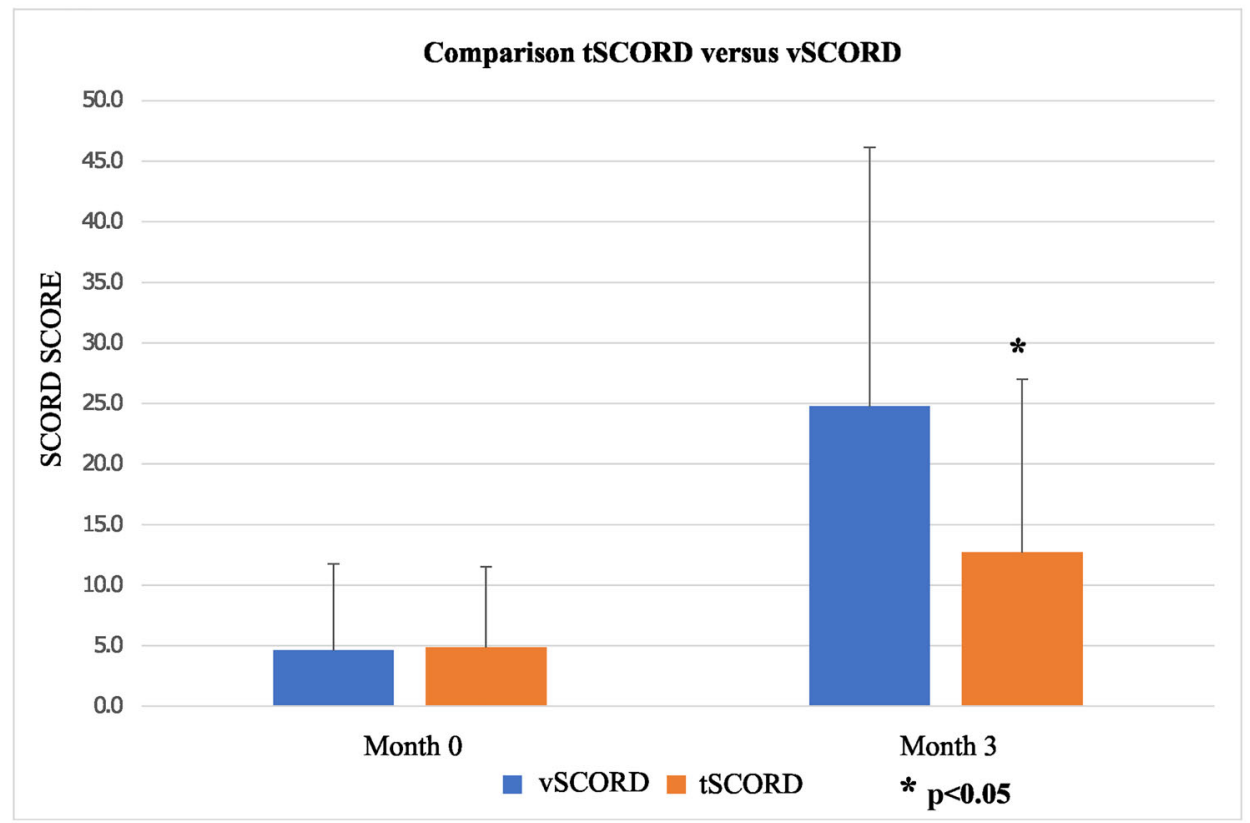

Fig. 2 A Mechlorethamine (MCN) gel monotherapy shows increased dermatitis as measured by the SCORD score. The ratio of tSCORD (triamcinolone (TAC) plus MCN gel therapy) compared to vSCORD (MCN gel

patients with mild, moderate, or severe dermatitis, there was maximal benefit of triamcinolone in lesions with mild and moderate dermatitis, particularly in months 2 and 3 of treatment (i.e., in patients with the most severe dermatitis, triamcinolone did not significantly monotherapy) shows that dermatitis is decreased in all months of study treatment. B At the peak level of dermatitis, in month 3 , addition of TAC significantly decreases dermatitis

help control dermatitis). Therefore, contact dermatitis was decreased at all time points in the study by adding triamcinolone.

There was a statistically significant difference in pruritus between lesions in patients with severe dermatitis at 3 months who were 
receiving triamcinolone $\left(\mathrm{VAS}_{\mathrm{avg}}=2.03\right)$ compared to mechlorethamine/chlormethine gel alone $\left(\mathrm{VAS}_{\mathrm{avg}}=4.93\right)$.

\section{Patch Testing}

Patients who developed severe contact dermatitis, defined as blistering, severe pain, or requiring interruption or discontinuation of therapy were biopsied and patch tested on normal-appearing skin. A $2+$ reaction at the 96-h reading was observed in 10 of 12 (83.3\%) patients. Of these 10 patients, 8 also showed reactions to T.R.U.E. Test antigens. A total of 5 of 12 patients $(41.6 \%)$ showed a reaction to the vehicle alone and none of these were found to react to $20 \%$ propylene glycol or $1 \%$ menthol (components of the vehicle). Two of 12 (16.6\%) patients showed no reaction (i.e., an irritanttype reaction), despite having clinical dermatitis and an elevated SCORD score.

\section{Dermatopathology}

Blinded dermatopathologic evaluation of all treatment site biopsies was performed. Little difference between the two arms was noted in biopsies of patients' lymphoma with the exception of one case where the dermatitis was more prominent in the mechlorethamine/ chlormethine gel only arm. This is likely because the second biopsy was performed 1 month after treatment discontinuation (i.e., 5 months). The biopsies of non-lesional patch test sites compared the vehicle control or baseline biopsy with the $96-\mathrm{h}$ patch test biopsy. The two patients with irritant contact dermatitis showed minimal dermatitis. However, most of the 10 patients with a $2+$ reaction showed a similar pattern of inflammation. Unlike a classic allergic contact dermatitis pattern with spongiotic vesicles and a predominantly superficial infiltrate, these patients showed papillary dermal edema and a more prominent inflammatory infiltrate involving both the superficial and deep dermis, reminiscent of an arthropod assault reaction (Fig. 3).

\section{CAILS}

The composite assessment of index lesions (CAILS) score was measured to assess therapeutic impact of coadministration of triamcinolone ointment with mechlorethamine/chlormethine gel. Figure $4 \mathrm{~A}$ shows that the addition of triamcinolone ointment does not diminish the therapeutic effects of mechlorethamine/ chlormethine gel. While not statistically significant, there was some improvement in CAILs in the combined treatment arm compared to the mechlorethamine/chlormethine gel alone. Because both topical corticosteroids and mechlorethamine/chlormethine gel individually have benefit in treatment of MF $[6,19]$, there may be some additive benefit from coadministration of both. Further, in a subgroup analysis of both study arms, there was no decrease in CAILs scoring among patients with severe or moderate contact dermatitis. This suggests that severe dermatitis is not associated with better response to therapy.

The molecular identity of $\mathrm{T}$ cell clones was identified in pre- and post-treatment sites. Figure $4 \mathrm{~B}$ shows a representative patient who had three individual malignant clones identified at baseline. This patient had a clinical response and an improved CAILs score with therapy. In each of the two study arms, the malignant clones were decreased at follow-up compared to the baseline skin biopsy. In all patients who developed contact dermatitis, there was no expansion of baseline malignant clones during the contact dermatitis flare (data not shown).

\section{DISCUSSION}

Despite strong data supporting the efficacy of topical mechlorethamine/chlormethine and easier access to a drug with FDA approval in the treatment of MF, it remains underutilized [5]. In the present study we help define the problem of contact dermatitis and identify that triamcinolone $0.1 \%$ ointment can be helpful at decreasing this most common adverse reaction without affecting mechlorethamine/chlormethine efficacy. 

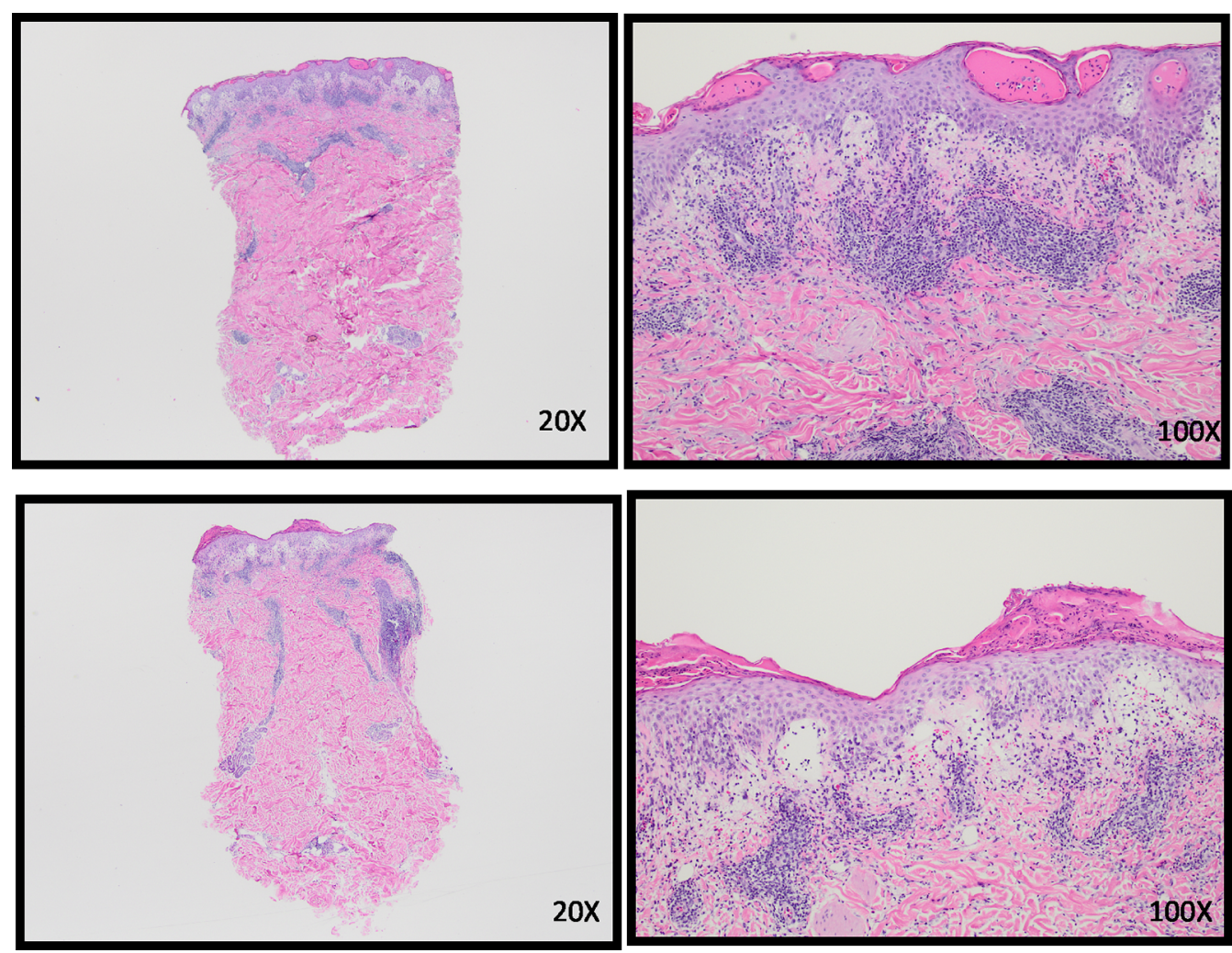

Fig. 3 Mechlorethamine induced contact dermatitis (patient 21 and 23): acute spongiosis with papillary dermal edema. Brisk superficial and deep perivascular infiltrates with eosinophils

The peak of adverse reactions occurs at months 2 and 3 and lesions treated with triamcinolone had decreased contact dermatitis. Patients who had more contact dermatitis and higher SCORD scores were more likely to require an interruption or discontinuation of topical mechlorethamine/chlormethine gel treatment. Quality of life was improved while on triamcinolone, as itch was significantly decreased.

There were no clear predisposing factors to predict who would develop contact dermatitis with mechlorethamine/chlormethine therapy. It occurred in both sexes, both stages (IA and IB), and in patients with patches and plaques (data not shown). Most patients with severe contact dermatitis reactions were found to react to unrelated antigens on T.R.U.E. Test, possibly suggesting an allergic diathesis. Most patients, however, had no history of skin atopy or seasonal allergies. Additionally, skin pathology findings were not that of standard allergic contact dermatitis, and it remains unclear whether the most common mechlorethamine/ chlormethine reactions are true type IV delayed hypersensitivity responses. Within the same patient, we observed that some lesions never develop contact dermatitis while others do, and some patients can restart mechlorethamine/ chlormethine despite an initial brisk clinical reaction, arguing against a classical allergic contact dermatitis that would require discontinuation and avoidance. Thus, many individuals experiencing a cutaneous reaction on topical mechlorethamine/chlormethine may be able to restart therapy after a pause.

Finally, the coadministration of triamcinolone $0.1 \%$ ointment and mechlorethamine/ chlormethine gel does not adversely affect the efficacy of this therapy. One hypothetical mechanism of action for the benefit of topical mechlorethamine/chlormethine is inducing inflammation and an antitumor response [20]. If triamcinolone were to dampen this 
A.

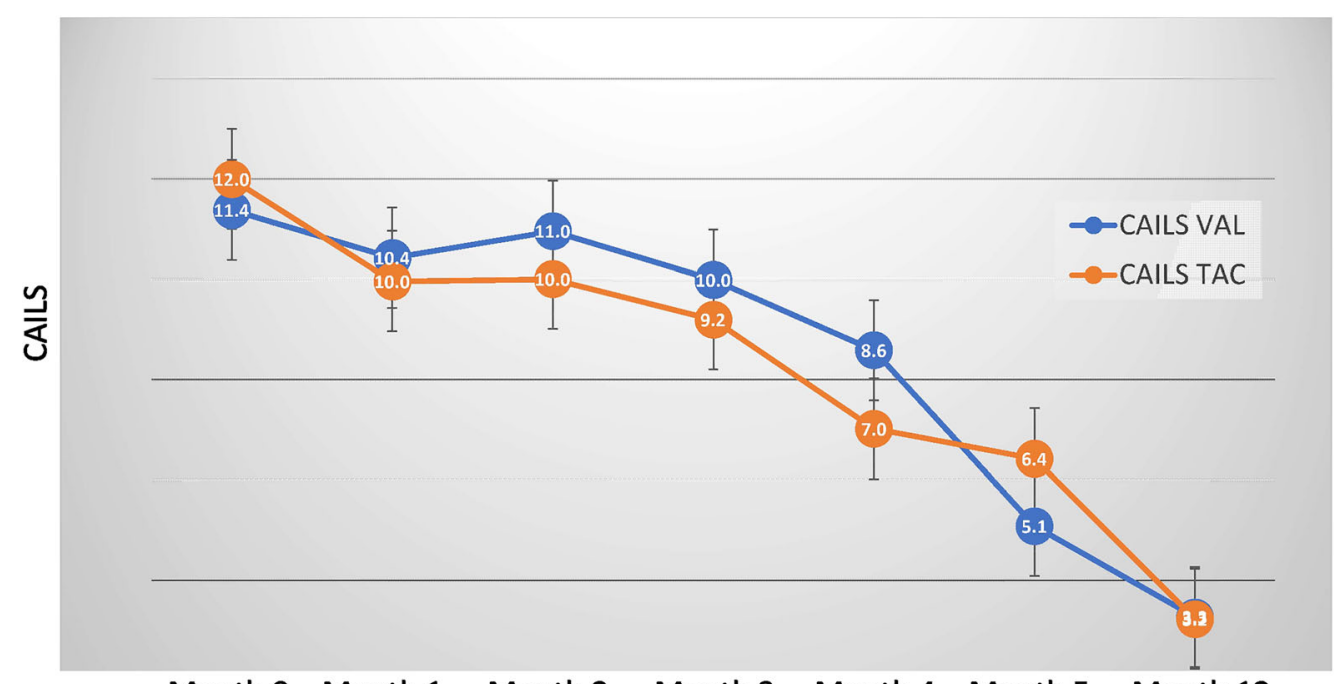

Month 0 Month 1 Month 2 Month 3 Month 4 Month 5 Month 12

B.

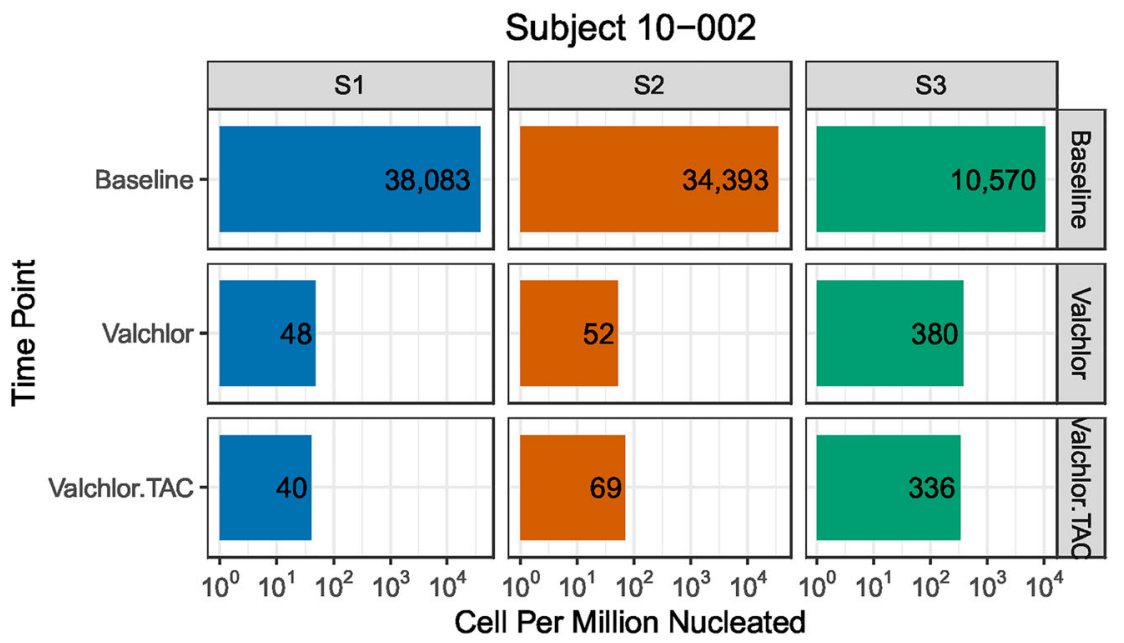

Fig. 4 Addition of triamcinolone (TAC) $0.01 \%$ ointment to mechlorethamine $(\mathrm{MCN})$ gel therapy does not affect efficacy. A Treatment with MCN gel alone (CAILS VAL) versus MCN gel and TAC ointment (CAILS TAC) result in similar composite assessment (CAILS) scores. B Three

inflammation and possibly antitumor response, this could lead to a diminished treatment response. There was no decrease in CAILs scores in the triamcinolone + mechlorethamine/ chlormethine arm compared to the mechlorethamine/chlormethine arm alone. Additionally, there was no increase in number of malignant clones in any patient who developed contact dermatitis, suggesting that the immune
$\mathrm{T}$ cell clones in the skin identified at baseline are treated with MCN gel alone (Valchlor) or combined treatment with MCN and TAC (Valchlor.TAC) are significantly diminished at month 5

response driving the contact dermatitis was distinct from the lymphoma. It is highly unlikely that inflammation from contact dermatitis flares will result in worsening lymphoma. Lastly, the study design allowed each patient to act as his own control. Topical triamcinolone could potentially cause systemic actions through absorption. Nevertheless, this would tend to diminish the differences between the 
two treatment arms that were observed rather than contradict the outcome. Moreover, previous studies have shown no notable adrenal suppression or systemic effects with even greater levels of triamcinolone application (which in this study were limited to plaques that generally only covered a few percent of the body surface area) [21].

This study provides strong evidence that coadministration of triamcinolone $0.1 \%$ ointment with mechlorethamine/chlormethine gel is an effective and safe therapeutic approach to treatment of MF. By minimizing the contact dermatitis associated with mechlorethamine/ chlormethine gel treatment and improving tolerability, this efficacious therapy for MF should be more widely considered.

\section{Limitations}

This study was limited to one center and only included patients who were willing to participate in the study (e.g., willing to perform monthly visits for $5+1$ months). While patients' lesions were randomized to treatment, each patient received both therapies and therefore this was not blinded. Patients were their own control and while there was insufficient topical triamcinolone applied to create systemic exposure and measured lesions were separate, this did lead to one patient mixing up the treatment arms from days 1 to 30 (he was excluded from analysis except for intention to treat analysis). The study has a small sample size. Use of an ultrapotent topical steroid or more frequent steroid dosing may have had a greater reduction in contact dermatitis.

\section{CONCLUSIONS}

Early-stage mycosis fungoides requires skin-directed therapies that are safe and effective. Topical mechlorethamine/chlormethine has been a proved effective therapy but contact dermatitis can limit its use. The MIDAS study establishes that peak dermatitis severity occurs after 2 to 3 months of topical $\mathrm{MCN}$ in most patients, and this is significantly decreased by coadministration of topical triamcinolone. This should allow patients to stay on therapy for longer owing to the improved side effect profile.

\section{ACKNOWLEDGEMENTS}

Funding. This randomized controlled trial was supported by Helsinn and the Rochester General Hospital Skin Disease Research Fund. The journal's Rapid Service Fee was funded by Helsinn.

Authorship. All named authors meet the International Committee of Medical Journal Editors (ICMJE) criteria for authorship for this article, take responsibility for the integrity of the work as a whole, and have given their approval for this version to be published."

Author Contributions. CVA, ESG, and BP were involved in all aspects of the study from design through manuscript writing. CGC performed dermatopathology and assisted in writing the manuscript. SMC performed statistical analysis and revising of the manuscript. CVA is now a Dermatology Fellow at the University of North Carolina School of Medicine. Chapel Hill, NC USA.

Disclosures. Carolina V. Alexander-Savino, Catherine G. Chung, Elaine S. Gilmore, Sean M. Carroll, Brian Poligone have nothing to disclose.

Compliance with Ethics Guidelines. This was a prospective, randomized, controlled study approved by the WCG Institution Review Board. This study was performed in accordance with the Helsinki Declaration of 1964 and its later amendments. All patients voluntarily signed an informed consent form (ICF) that was approved by the Western Institutional Review Board (WIRB). The ICF was delivered verbally by the investigator and/or clinical coordinator and all questions answered by the investigator or coordinator. Consent was provided before any study-related procedures were performed. 
Data Availability. Except when legally prohibited or because of privacy laws, the datasets generated during and/or analyzed during the current study are available from the corresponding author on reasonable request.

Open Access. This article is licensed under a Creative Commons Attribution-NonCommercial 4.0 International License, which permits any non-commercial use, sharing, adaptation, distribution and reproduction in any medium or format, as long as you give appropriate credit to the original author(s) and the source, provide a link to the Creative Commons licence, and indicate if changes were made. The images or other third party material in this article are included in the article's Creative Commons licence, unless indicated otherwise in a credit line to the material. If material is not included in the article's Creative Commons licence and your intended use is not permitted by statutory regulation or exceeds the permitted use, you will need to obtain permission directly from the copyright holder. To view a copy of this licence, visit http://creativecommons.org/licenses/by$\mathrm{nc} / 4.0 /$.

\section{REFERENCES}

1. Pimpinelli N, Olsen EA, Santucci M, et al. Defining early mycosis fungoides. J Am Acad Dermatol. 2005;53(6):1053-63.

2. Mehta-Shah N, Horwitz SM, Ansell S, et al. NCCN Guidelines Insights: Primary Cutaneous Lymphomas, Version 1.2021. J Natl Compr Cancer Netw. 2021.

3. Trautinger F, Eder J, Assaf C, et al. European Organisation for Research and Treatment of Cancer consensus recommendations for the treatment of mycosis fungoides/Sezary syndrome; Update 2017. Eur J Cancer [Internet]. 2017;77:57-74. https://doi. org/10.1016/j.ejca.2017.02.027.

4. Willemze R, Hodak E, Zinzani PL, Specht L, Ladetto M. Primary cutaneous lymphomas: ESMO Clinical Practice Guidelines for diagnosis, treatment and follow-up. Ann Oncol [Internet]. 2018;29:iv30-40. https://doi.org/10.1093/annonc/mdy133.
5. Querfeld C, Pacheco T, Haverkos B, et al. A Little Experience Goes a Long Way: Chlormethine/ Mechlorethamine Treatment Duration as a Function of Clinician-level Patient Volume for Mycosis Fungoides Cutaneous T-cell Lymphoma (MF-CTCL) - a Retrospective Cohort Study. Front Med [Internet]. 2021;8:921 https://www.frontiersin.org/ article/10.3389/fmed.2021.679294.

6. Lessin SR, Duvic M, Guitart J, et al. Topical chemotherapy in cutaneous T-cell lymphoma: positive results of a randomized, controlled, multicenter trial testing the efficacy and safety of a novel mechlorethamine, $0.02 \%$, gel in mycosis fungoides. JAMA Dermatol [Internet]. 2013;149(1):25-32. http://www.ncbi.nlm.nih.gov/pubmed/23069814.

7. Krumbhaar EB. Role of the blood and the bone marrow in certain forms of gas poisoning. J Am Med Assoc. 1919.

8. Haserick JR, Richardson JH, Grant DJ. Remission of lesions in mycosis fungoides following topical application of nitrogen mustard: a case report. Cleve Clin J Med. 1959.

9. Van Scott EJ, Winters PL. Responses of mycosis fungoides to intensive external treatment with nitrogen mustard. Arch Dermatol. 1970.

10. Kim YH, Martinez G, Varghese A, Hoppe RT. Topical nitrogen mustard in the management of mycosis fungoides: update of the Stanford experience. Arch Dermatol. 2003;139(2):165-73.

11. Price NM, Constantine VS, Hoppe RT, Fuks ZY, Farber EM. Topical mechlorethamine therapy for mycosis fungoides. Br J Dermatol. 1977;97(5): 547-50.

12. Price NM, Hoppe RT, Deneau DG. Ointment-based mechlorethamine treatment for mycosis fungoides. Cancer. 1983.

13. Ramsay DL, Parnes RE, Dubin N. Response of mycosis fungoides to topical chemotherapy with mechlorethamine. JAMA Dermatology [Internet]. 1984;120(12):1585-90. https://doi.org/10.1001/ archderm.1984.01650480047016.

14. Esteve E, Bagot M, Joly P, et al. A prospective study of cutaneous intolerance to topical mechlorethamine therapy in patients with cutaneous T-cell lymphomas. French Study Group of Cutaneous Lymphomas. Arch Dermatol [Internet]. 1999;135(11):1349-53. http://www.ncbi.nlm.nih. gov/pubmed/10566833.

15. De Quatrebarbes J, Estève E, Bagot M, et al. Treatment of early-stage mycosis fungoides with twiceweekly applications of mechlorethamine and 
topical corticosteroids: a prospective study. Arch Dermatol. 2005.

16. Van Scott EJ. Complete regressions of mycosis fungoides with topical mechlorethamine hydrochloride. JAMA [Internet]. 1972;222(9):1172. https://doi.org/10.1001/jama.1972. 03210090052026.

17. Ramsay DL, Halperin PS, Zeleniuch-Jacquotte A. Topical mechlorethamine therapy for early stage mycosis fungoides. J Am Acad Dermatol. 1988;19(4):684-91.

18. Kunz B, Oranje AP, Labrèze L, Stalder JF, Ring J, Taïeb A. Clinical validation and guidelines for the SCORAD index: consensus report of the European Task Force on Atopic Dermatitis. Dermatology. 1997;195(1):10-9.
19. Kartan S, Shalabi D, O’Donnell M, et al. Response to topical corticosteroid monotherapy in mycosis fungoides. J Am Acad Dermatol [Internet]. 2021;84(3):615-23. https://doi.org/10.1016/j.jaad. 2020.05 .043 .

20. Vonderheid EC, Ekbote SK, Kerrigan K, et al. The prognostic significance of delayed hypersensitivity to dinitrochlorobenzene and mechlorethamine hydrochloride in cutaneous $\mathrm{T}$ cell lymphoma. J Invest Dermatol. 1998;110(6):946-50.

21. Rasmussen JE. Percutaneous absorption of topically applied triamcinolone in children. Arch Dermatol [Internet]. 1978;114(8):1165-7. https://doi.org/10. 1001/archderm.1978.01640200019005. 